\title{
Sexually Transmitted Infections
}

\section{Editorials}

\section{South Africa: host to a new and emerging HIV epidemic}

Southern Africa is experiencing a rapidly growing HIV epidemic, and this small region currently accounts for a disproportionately large segment of the global burden of new HIV infections. ${ }^{1}$ In this region, HIV is spreading predominantly through heterosexual contact and clade $\mathrm{C}$ is the predominant subtype. In 1996, the reported HIV prevalence among antenatal clinic attenders from the southern African countries of Zimbabwe, Malawi, and Botswana were $40 \%, 30 \%$, and $30 \%$ respectively. $^{2}$

Before 1987 HIV infection in South Africa was rare. ${ }^{3}$ Annual, anonymous, national HIV seroprevalence surveys conducted among first time antenatal clinic attenders provide an indication of the emergence and trends in the progression of HIV infection in South Africa. In the past 10 years HIV seroprevalence has risen from $0.76 \%$ in 1990 to $10.44 \%$ in 1995 to $22.8 \%$ in $1998,{ }^{4}$ with no signs of having reached a plateau. It is estimated that there are currently 3.5 million South Africans infected with HIV. This rapidly growing HIV epidemic in South Africa is best described as explosive.

An HIV incidence of $11.9 \%$ in 1997 in women between the ages of 15 and 30 years derived from repeat antenatal seroprevalence surveys (each with a sample size of approximately 1000) conducted in Hlabisa, a rural community on the east coast of South Africa, from 1992 to 1997, illustrates the explosive nature of the HIV epidemic. ${ }^{5}$ Even in the relatively low incidence year of 1993, 3.8\% of HIV negative women became infected. Young women in the general population in South Africa are experiencing HIV infection rates previously seen only in high risk sex worker populations. ${ }^{67} \mathrm{HIV}$ is spreading most rapidly in young women in South Africa; HIV prevalence grew from $6.9 \%$ in 1992 to $21.1 \%$ in 1995 in the $20-24$ year old age group,${ }^{8}$ highlighting the importance of youth in the South African HIV epidemic.

Community based, HIV seroprevalence surveys conducted in one rural area of South Africa in 1990 and 1992 demonstrated that HIV infection was four times more prevalent among women $(1.6 \%)$ than men $(0.4 \%)$ and that women become infected at an earlier age than men. ${ }^{9} \mathrm{~A}$ repeat survey in 1994 demonstrated a 2.3-fold sex difference in HIV prevalence, showing a narrowing of the sex gap as the epidemic progresses. ${ }^{10}$

Migrant labour is a major factor in the spread of HIV and other sexually transmitted diseases in South Africa. Despite the demise of apartheid, migration is still part of the reality of many South African lives. A woman's risk of $\mathrm{HIV}$ infection is substantially increased if her partner is a migrant worker. A study from rural South Africa found that women whose partners spent 10 or fewer nights per month at home had an HIV prevalence of $13.7 \%$ compared with $0 \%$ in women who spent more than 10 nights in a month with their partners. ${ }^{10}$ Using crude measurements of mobility/migration, population based surveys from rural KwaZulu-Natal found about 2.5 times more infections among mobile adults compared with adults resident in the area continuously for more than a year. ${ }^{9}$ Among women, migration was associated with an age adjusted 2.4-fold higher risk of HIV infection compared with a 7.3-fold higher risk among men. ${ }^{9}$ The patterns of migration and sexual networking are fairly complex and implications for the spread of HIV and other sexually transmitted diseases are elaborated further in a paper by Lurie et al. ${ }^{11}$

The associated epidemic of sexually transmitted infections (STIs) is also a major contributor to the burden of disease in South Africa. On any given day about one in every four of the approximately 60000 women aged 15-49 years in Hlabisa is infected with at least one STI. ${ }^{12}$ Of these, $48 \%$ are asymptomatic, $50 \%$ are symptomatic but not seeking care, and only $2 \%$ seek care during an illness episode. Of these handful of women who seek care, only two out of three women are adequately treated for the STI. ${ }^{12} \mathrm{~A}$ policy of syndromic management of STIs as well as the integration of STI services into the general primary healthcare services, adopted in 1995, have been steps in the right direction in terms of managing the huge burden of STIs; however, its success at the health facilities level has been impeded by poor drug supplies, low condom use, inability to encourage partner referral for treatment, and the social stigma associated with seeking STI treatment.

The most common HIV/AIDS presenting opportunistic infection in South Africa is tuberculosis. The progression from asymptomatic HIV to early disease is best reflected in the rise in new tuberculosis cases and the number of co-infections with HIV. New tuberculosis cases have a similar age and sex profile to that seen in the HIV epidemic. In Hlabisa, co-infection with HIV in adult tuberculosis rose from $36 \%$ in 1993 to $59 \%$ in 1995 and $65 \%$ in $1997 .^{13}$

What makes the South African HIV epidemic a new and emerging explosive epidemic? Compared with other countries in eastern and central Africa, HIV infection in South Africa is a new phenomenon. Despite the late introduction of the virus it has been experiencing an intensely rapid growth and progression of the HIV epidemic. Having already reached high levels of infection there is no indication of stabilisation or plateauing of the epidemic. The migrant labour system and high levels of other sexually transmitted diseases are factors that are enhancing the transmission of HIV and fuelling this explosive epidemic. Of concern is the high HIV incidence rates in young people, and young women in particular. 
The relatively late introduction of HIV into South Africa provided an opportunity to establish prevention programmes at an early stage. However, this opportunity was lost through the inability and lack of credibility of the previous government to institute any meaningful interventions. The new, democratically elected government while committed to addressing the HIV epidemic has to date also been unable to mount a response of the scale and magnitude required to turn this epidemic around. The current state of the HIV epidemic in South Africa poses many challenges. The continued and explosively rapid spread of HIV can be reduced through strategic and decisive action. Significantly, and in the medium to long term, how to reduce the impact of migration on HIV transmission and improve the status of women in the face of a bleak economic future and current high unemployment rates are urgent questions. While the focus must continue to be on preventing new HIV infections, strategies to deal with the increasing burden of HIV related diseases and AIDS as well as the impact of the premature loss of lives must also be developed. The success of these strategies will depend on the ability to develop effective partnerships between government, civil society, private and nongovernmental sectors.

Q ABDOOL KARIM

Centre for Epidemiological Research in South Africa, South African Medical Research Council and Southern African Fogarty HIVIAIDS International Training and Research Programme, South Africa
S S ABDOOL KARIM

Centre for Epidemiological Research in South Africa, South African

Medical Research Council, South Africa and Division of Epidemiology,

Columbia University, New York, USA

1 Joint United Nations Programme on HIV/AIDS and the World Health Organisation. Report on the status of the HIVIAIDS pandemic. Geneva: UNAIDS and WHO, 1998.

2 US Bureau of Census. Metropolitan Life Doyle Model. Business Day August 1997.

Abdool Karim SS, Abdool Karim Q. Changes in HIV seroprevalence in a rural black community in KwaZulu (letter). S Afr Med f 1992;82:484.

4 Department of Health, RSA. 1998 National HIV sero-prevalence survey of women attending antenatal clinics in South Africa. Summary report. Pretoria: Health Systems Research and Epidemiology, DOH, 1999.

5 Abdool Karim SS. Establishing a South African vaccine trial site through intervention trials of HIV prevention among high risk rural female sexual partners of migrant workers in Hlabisa. HIVNET proposal submitted to Family Health International, April 1997.

6 Nzila N, Laga M, Thaim MA, et al. HIV and other sexually transmitted diseases among female prostitutes in Kinshasa. AIDS 1991;5:715-21.

7 Ramjee G, Abdool Karim SS, Sturm AW. Sexually transmitted infections among sex workers in KwaZulu-Natal, South Africa. Sex Transm Dis 1998; 25:346-9.

8 Coleman RL, Wilkinson D. Increasing HIV prevalence in a rural district of South Africa. F Acquir Immun Def Syndr Hum Retrovirol 1997;16:50-3.

9 Abdool Karim Q, Abdool Karim SS, Singh B, et al. HIV infection in rural South Africa. AIDS 1992;6:1535-9.

10 Colvin M, Abdool Karim SS, Connolly C, et al. HIV infection and asymptomatic sexually transmitted infections in a rural South African community. Int F STD AIDS 1998;9:548-50.

11 Lurie M, Harrison A, Wilkinson D, et al. Circular migration and sexual networking in rural KwaZulu/Natal: implications for the spread of HIV and other sexually transmitted diseases. Health Trans Rev 1997:15-24.

12 Wilkinson D, Abdool Karim SS, Harrison A, et al. Unrecognised sexually transmitted infections in rural South African Women-the hidden epidemic. Bull World Health Organ 1999; (in press).

13 Wilkinson D. TB research in South Africa. S Afr Med f 1999;89:155-9.

\section{The use of HIV resistance assays - random or randomised?}

High frequency error prone replication is the mechanism by which HIV-1 exhibits Darwinian evolution of its reverse transcriptase and protease genes in a changing drug environment and which may be one of the causes of treatment failure. ${ }^{1}$ Genotypic and phenotypic resistance assays have been developed to evaluate this evolution of drug resistant virus and many clinicians and virologists consider that there is sufficient research based knowledge to introduce these assays into the routine clinical care of individuals with HIV-1 infection. ${ }^{2}$ This process will take time and access is likely to be inequitable at the outset, depending largely on the response of fund holders to requests for funding. Purchasers will rightly be reluctant to provide resources for health interventions until there is evidence of their clinical value and, increasingly, their cost effectiveness. How strong is this evidence at present for HIV resistance testing?

In vitro, HIV-1 grown in the presence of one or more antiretroviral drugs will rapidly evolve mutations that are associated with loss of sensitivity to the drug. ${ }^{3}$ In vivo, early studies showed that loss of virological suppression with monotherapies was associated with evolution of genotypic and phenotypic resistance. ${ }^{4}$ Retrospective analysis of stored specimens of subgroups of patients in clinical trials suggested that resistance at baseline or evolving during therapy was associated with clinical failure..$^{56}$

The rationale for resistance testing is to optimise therapy, particularly when drugs are being changed following virological failure. Instead of changing all components of a regimen, resistance testing should allow continuation of drugs to which the virus is still sensitive, thereby preserving a wider range of therapeutic options for the future. However, a number of important factors underlie this simple hypothesis. ${ }^{1}$
(1) Although several studies have demonstrated a strong association between the presence of certain mutations and phenotypic drug resistance, ${ }^{7}$ our knowledge of the association is incomplete and will always remain so, particularly when drugs are used in combination as they are now. In addition, the link between resistance, particularly genotypic, and therapeutic failure is less clearly established for some drug classes. ${ }^{8}$

(2) The interpretation of genotypic resistance results is highly complex, particularly when drugs are used in combination as they are now. More research is needed to develop and validate algorithms to interpret results, which will be an integral part of clinical guidelines, and on the best way to deliver advice to clinicians. However, it is unlikely that resistance tests will ever be able to predict in vivo activity of individual drugs with complete accuracy.

(3) Current genotypic and phenotypic assays both have their limitations in a clinical setting: they require 1000-2000 HIV-1 RNA copies per $\mathrm{ml}$ of plasma from specimens collected from patients while on drug therapy to measure resistance reliably and are unable to detect minority species. High throughput phenotypic assays are expensive and are only performed in specialist units (none in the United Kingdom).

(4) Treatment failure is not always the result of resistance; other important factors include non-adherence, individual pharmacokinetics, and drug interactions.

Information on the predictive value of genotypic and/or phenotypic results for the selection of new regimens in patients who are failing therapy is very limited. Two small prospective randomised controlled trials have explored the use of resistance assays in highly treatment experienced individuals failing therapy and provide some evidence to support its use. $^{910}$ The VIRADAPT and CPCRA 046 
(GART) trials found that the patients allocated to have a genotypic resistance test had a significantly greater reduction in viral load than those who did not (mean differences of $0.8 \log _{10}$ copies per $\mathrm{ml}$ at 6 months for VIRADAPT and $0.6 \log _{10}$ copies per $\mathrm{ml}$ at $4-8$ weeks for CPCRA 046). Different algorithms were used to interpret the mutations detected in the two studies. These studies require caution in their interpretation as follow up was short and, as they included individuals with relatively advanced HIV infection and considerable previous anti-HIV therapy, the findings cannot be directly extrapolated to "naive" or less experienced individuals. There were also differences in the management of the randomised groups, apart from the access to resistance assays, in the level of expert review provided to the clinicians. Clearly, further randomised controlled trials are required to evaluate the long term impact of resistance testing.

The simplest part of the cost effectiveness equation is the cost. Approximately 7500 patients - that is, half of the HIV-1 infected individuals in England and Wales, currently receive antiretroviral therapy - a figure likely to double over the next 5 years. ${ }^{112}$ Gene sequencing, the most likely first line technology, at about $£ 250$ per test will cost approximately $£ 1.9$ million per annum for one test per year (approximately 3\% of the annual HIV/AIDS drug budget). In addition, the input required from clinical virologists to interpret the tests and provide advice on a day to day basis will be significant and costs for plasma sample handling must be considered. However, this would be a small price to pay for a test if it has an appreciable impact upon the magnitude and durability of response to therapy. Phenotyping would approximately double the cost of testing but might have greater benefits.

We are in a period of evolving evidence for the role of resistance testing in clinical care. However, pressure from patients, clinicians, and virologists (individually and as corporate bodies) is likely to lead to the introduction of testing in clinical practice in the near future. This should not be allowed to occur at random. We have a responsibility to determine in what patient population resistance testing is most useful and cost effective and which tests to use. There is a narrow window of opportunity in the United Kingdom to conduct studies of how resistance testing should be used and which methodologies should be employed. It would be unfortunate if this window were to close before we have had an opportunity to resolve at least some of these issues.

CLIVE LOVEDAY Department of Retrovirology, Royal Free and University College Medical School, London

DAVID DUNN

SHEENA MCCORMACK ABDEL BABIKER

HIV Division, Medical Research Council Clinical Trials Unit

1 Hirsch MS, Conway B, D'Aquila RT, et al. Antiretroviral drug resistance testing in adults with HIV infection. Implications for clinical management. AMA 1998;279:1984-90

2 AIDS Treatment Project Dr Fax. Resistance Assays in Clinical Practice. October 1998.

3 Larder BA, Kemp SD. Multiple mutations in HIV-1 reverse transcriptase confer high-level resistance to zidovudine. Science 1989;246:1155-8.

4 Rey D, Hughes M, Pi JT, et al. HIV-1 reverse transcriptase codon 215 mutation in plasam RNA: immunologic and virologic responses to zidovudine. The AIDS Clinical Trials Group Study 175 Virology Team. F Acquir Immune Defic Syndr Hum Retrovirol 1998;17:203-8.

5 Japour AJ, Welles S, D'Aquila RT, et al. Prevalence and clinical significance of zidovudine resistance mutations in human immunodeficiency virus isolated from patients after long-term zidovudine therapy. AIDS Clinical Trials Group $116 \mathrm{~B} / 117$ Study Team and the Virology Committee Resistance Working Group. F Infect Dis 1995;171:1172-9.

6 Montaner JSG, Singer S, Schechter MT, et al. Clinical correlates of in vitro HIV-resistance to zidovudine. Results of the Multicentre Canadian AZT Trial. AIDS 1993;7:189-96.

7 Teddar RS, Kaye S, Loveday C, et al. Comparison of culture and non-culture-based methods for quantification of viral load and resistance to antiretroviral drugs in patients given zidovudine monotherapy. $f$ Clin Microbiol 1998;36:1056-63.

8 Condra JH, Holder DJ, Graham DJ, et al. Genotypic or phenotypic resistance susceptibility testing may not predict clinical responses to indinavir. Antiviral Ther 1997;2(suppl 5):abstract 47.

9 Durant J, Clevenburgh P, Halfon P, et al. Can HIV genotype determination be useful for individualised adaption of antiretroviral therapy? The French VIRADAPT Study. AIDS 1998;12(suppl 4):S16, OP 7.1.

10 Baxter JD, Mayers DL, Wentworth DN, et al. A pilot study of the short-term effects of antiretroviral management based on plasma genotypic antiretroviral resistance testing (GART) in patients failing antiretroviral therapy. 6th Conference on Retroviruses and Opportunistic Infections, Chicago, 1999 (LB8).

11 Molesworth A. Results from the 1995 survey of prevalent clinically diagnosed HIV infection in England and Wales. Commune Dis Rep 1997; R77-82.

12 Aalen OO, Farewell VT, De Angelis D, et al. New therapy explains the fall in AIDS incidence with a substantial rise in the number of persons on treatment expected. AIDS 1999;13:103-8.

\section{Commentary: Resistance testing — a clinical perspective}

The practicality and utility of performing prospective, randomised controlled trials (RCT) for HIV viral resistance testing is currently under debate within the HIV caring community. It is a truism that all new diagnostic tests should be scientifically and prospectively evaluated before they are introduced into clinical practice. However, in reality this rarely happens. The reasons for this are complex but include a belief that more information about a patient's clinical state is bound to be of benefit. For example, Mycobacterium tuberculosis resistance testing was prospective evaluated and showed little benefit. ${ }^{1}$ However, these tests continue to be widely performed! If RCTs were to show that HIV resistance testing was not of benefit, this would be unlikely to prevent their introduction. Indeed, many virologists and physicians would question the trial design, testing methodology, test interpretation, and drug choice.

Cost effectiveness arguments have been raised to suggest that RCTs of HIV resistance testing are important. In reality, the cost of drugs to treat HIV patients is so high that even modest reductions in the number of drugs included in a combination, which do not contribute to therapeutic effect, are likely to be cost effective.
The two randomised prospective studies of utilisation of resistance testing quoted in the editorial by Loveday and colleagues above indicate a modest benefit in nucleoside analogue/protease inhibitor experienced patients. ${ }^{2}{ }^{3}$ The follow up in these two small studies was short. In clinical practice, those who respond poorly to a particular regimen are likely to have their therapy changed and thus long term follow up is required to prove that reductions in HIV viral load achieved as a result of modifying antiretroviral therapy, according to a resistance test, are better sustained in those groups that have received resistance testing.

We are not arguing for no further research into the utility of resistance testing but that the value of an RCT would be limited as it may not address the research priorities or answer the question of whether resistance testing would be valuable in a given clinical situation.

\section{Research priorities}

The first priority is to ensure the information we obtain is accurate through good quality control systems.

Secondly, we need to be certain as to which is the optimal test in a particular circumstance. 
Thirdly, it remains ill defined as to whether most viral mutations with reduced sensitivity to a drug remain present in the circulation in sufficient quantities to be detected once the drug is removed. AZT resistant mutants do persist ${ }^{4}$ whereas levels of $3 \mathrm{TC}^{5}$ and possibly indinavir ${ }^{6}$ resistant strains reduce rapidly to potentially undetectable levels only to rapidly re-emerge following reintroduction of therapy.

Fourthly, one of the major difficulties of interpreting resistance testing is the importance that should be attached to common polymorphisms in the viral genome. Many of the research priorities require the use of large data bases in which virological responses to antiretroviral therapy in thousands of patients can be correlated with baseline resistance information rather than an $\mathrm{RCT}^{7}$

\section{Clinical uses of resistance testing}

The utility of performing resistance testing on virus derived from pregnant women or from individuals involved in accidental exposure to HIV is unlikely to be tested in randomised studies but will gain wide acceptance.

What is the utility of resistance testing before starting antiretroviral therapy for the first time? It has been established that resistant virus can be transmitted ${ }^{8}$ and this may be a reason for poor responses to therapy. We urgently need to gather more data on the prevalence of this problem. Most studies so far undertaken are small ${ }^{9}$ and, except in individuals in the process of seroconversion, may be misleadingly reassuring about the frequency of mutations. Assuming that resistant virus is sufficiently prevalent in treatment naive patients to make testing worthwhile, a rational approach at present might be to perform a line probe assay to detect AZT resistance and possibly 3TC resistance before therapy with close monitoring of viral load responses to initial treatment, with further resistance testing being performed in the patients taking the drugs who do not achieve the expected response.

The optimum algorithm of care following the failure of first treatment is unclear. If resistance to one agent only is shown, is it reasonable to change this agent only? If no resistance is shown, is it reasonable to intensify treatment with one drug or will the clinician feel that a complete change of therapy is most likely to produce a good clinical response? While the results of resistance testing may not influence treatment switches at this stage, it may prove important to know this when deciding what would be the optimum third or fourth line therapy. A resistance test should be performed, therefore, before the first regimen is discontinued. Whether the results are required at this time or whether the blood can simply be stored and analysed later is an open question.

Resistance testing is most likely to be used in the clinical setting in those patients who failed multiple previous therapies. A number of cohort studies and retrospective analyses attest to a benefit from knowledge of resistance testing in this situation. ${ }^{10}{ }^{11}$ Obviously there are multiple issues affecting virological responses to such regimens, including adherence, pharmacokinetic considerations, and replication in different body compartments, ${ }^{12}$ but it does seem likely that there is added value in knowing the results of a resistance test in addition to a detailed knowledge of the patient's previous drug history. Similar to antibiotic resistance testing this knowledge tells the clinician which drugs not to give rather than which drugs are likely to be optimum in therapy.

In conclusion, there is much that we do not know about the utility of resistance testing in improving the chances of a response to antiretroviral therapy. However, we believe the optimum way to improve our understanding is through careful case-control and large database studies. An RCT would have been appropriate 2 or 3 years ago; and this is an example of where rapid protocol development is crucial if the equipose, which is required both by the clinician and the client, is to be shared by sufficient numbers of individuals to make an RCT possible.

B G GAZZARD G MOYLE

Chelsea and Westminster Hospital, London

1 Hong Kong Tuberculosis Treatment Services, British Medical Research Council. A study in Hong Kong to evaluate the role of pretreatment susceptibility tests in the selection of regimens of chemotherapy for pulmonary tuberculosis-second report. Tubercule 1974;55:169-92

2 Durant J, Clevenburgh P, Halfon P, et al. Can HIV genotype determination be useful for individualised adaptation of antiretroviral therapy? The French VIRADAPT study. AIDS 1998;12 (suppl 4):S16, OP7.1.

3 Baxter JD, Mayers DL, Wentworth DN, et al, CPCRA 046 study team. A pilot study of the short-term effects of antiretroviral management based on plasma genotypic antiretroviral resistance testing (GART) in patients failing antiretroviral therapy. 6th Conference on Retroviruses and failing antiretroviral therapy. 6th Conference on Retroviruses
Opportunistic Infections, Chicago, 31 Jan-4 Feb 1999;abstract LB8.

4 Smith MS, Koerber KL, Pagano JS. Long-term persistence of zidovudine resistance mutations in plasma isolates of human immunodeficiency virus type 1 of dideoxyinosine-treated patients removed from zidovudine therapy. F Infect Dis 1994;169:184-8.

5 Kuritzkes DR, Quinn JB, Benoit SL, et al. Drug resistance and virologic response to NUCA3001, a randomized trial of lamivudine (3TC) versus zidovudine $(\mathrm{ZDV})$ versus $\mathrm{ZDV}$ plus3 TC in previously untreated patients. AIDS 1996;10:975-81.

6 Condra JH, Schlief WA, Blahy OM, et al. Dynamics of acquired HIV-1 clinical resistance to the protease inhibitor MK-639. 4th International HIV Drug-Resistance Workshop, Sardinia, 1995; Abstract 72.

7 Moyle GJ. Viral resistance patterns selected by antiretroviral drugs and their potential to guide treatment choice. Expert Opinion on Investigational Drugs 1997;6:943-64.

8 Erice A, Mayers DL, Strike DG, et al. Primary infection with zidovudineresistant human imunodeficiency virus type 1. N Engl f Med 1993;328: $1163-5$.

9 Angarano G, Monno L, Appice A, et al. Transmission of zidovudineresistant HIV-1 through heterosexual contacts. AIDS 1994;8:1013-4.

10 Zolopa AR, Shafer RW, Warford A, et al. Predictors of antiviral response to saquinavir/ritonavir therapy in a clinical cohort who have failed prior protease inhibitors: a comparison of clinical characteristics, antiretroviral drug history and HIV genotype. 2nd International Workshop on HIV Drug Resistance and Treatment Strategies, Lago Maggiore 24-27 June 1998:abstract 54 .

11 Larnier R, Danehower S, Daluge S, et al. Genotypic and phenotypic correlates of response to abacavir (ABC, 1592). 2nd International Workshop on HIV drug resistance and Treatment Strategies, Lago Maggiore 24-27 June 1998:abs resistance

12 Moyle GJ. Current knowledge of HIV-1 reverse transcriptase (RT) mutations selected during nucleoside analogue therapy: the potential to use resistance data to guide clinical decisions. F Antimicrob Chemother 1997;40 765-77.

\section{Should we be screening for asymptomatic HSV infections?}

Serological screening of population subgroups to detect HSV-2 infection is now being recommended as a disease control measure. ${ }^{12}$ The rationale for this is based on the emerging evidence of the contribution of subclinical viral shedding to herpes simplex virus (HSV) transmission, so that undiagnosed infections may be the major contributors to transmission at the population level. ${ }^{1} \mathrm{~A}$ case has been made for screening for HSV-2 in sexually transmitted disease (STD) clinic attenders, ${ }^{2}$ and in pregnant women to prevent neonatal herpes. ${ }^{1}$ This is supported by recent studies which showed a high level of willingness to accept a serological HSV-2 test among STD attenders $(90 \%)^{3}$ and antenatal clinic attenders $(80 \%) .{ }^{4}$ Several issues still need to be resolved; in particular, the existence of effective pre- 
ventive strategies; the acceptability and possible implications of screening for asymptomatic individuals; and the cost-benefit of screening as a control strategy in different populations.

A reduction of about $50 \%$ in the HSV-2 transmission rate per contact event, associated with the giving of counselling messages, has been reported for vaccine trial participants, ${ }^{5}$ many of whom are likely to be highly motivated individuals. However, in the absence of studies demonstrating effective risk reducing behaviour in population representative groups of HSV-2 infected individuals, including people outside stable relationships, one cannot yet conclude that knowledge of seropositive status will have a significant impact on subsequent sexual behaviour at the population level. Suppressive antiviral therapy significantly reduces viral shedding; however, its effectiveness in reducing transmission has not been established, and the results of further studies are awaited. ${ }^{2}$

Fairley and Monteiro reported a high level of acceptability of HSV serological screening among STD clinic attenders. $^{3}$ The merely marginal effect of a three page information sheet on the willingness of patients to undergo an HSV test is attributed by the authors to an "intuitive rather than a reasoned judgment." "The authors acknowledge that the study did not assess "patients' understanding of these complicated issues, the impact of counselling, or the time required to provide a level of understanding needed for informed consent." ${ }^{3}$ They conclude by adopting an appropriately cautious attitude, believing that "it is premature to make HSV type specific antibody tests widely available when costs may be high and benefit to patients uncertain." 3 The full costs of any screening programme, costs of subsequent patient management, and who will bear these costs need to be identified and measured. A prerequisite is to identify and measure the costs of adequate pretest and post-test counselling, which may need to be continued over a long period of time in the relatively large number of HSV-2 seropositives who would thereby be identified. The psychological costs of an unsolicited test and positive result for an asymptomatic person, in terms of impact on self esteem and subsequent psychosexual relationships, may well be high and should be taken into account. The cost of antiviral drugs to individual patients, and its negative impact on quality of life, is reported to be the most common reason for patients discontinuing treatment ${ }^{6}$; and long term suppressive therapy, should it be shown to be effective in preventing transmission, is unlikely to be acceptable to any but the most highly motivated individuals identified through such a programme.

Highly sensitive and specific type specific serological tests, when they become commercially available, are likely to be helpful as diagnostic tools in the clinical management of individuals with genital herpes disease. ${ }^{7}$ While potentially useful clinical aids for detecting atypical presentations, they may contribute to a different kind of clinical uncertainty in that atypical genital symptoms in a HSV-2 positive patient may not be caused by the HSV infection. The cost of a commercial type specific test, where there is high volume testing, has been estimated at $\$ 13(£ 8)$ per test. ${ }^{2}$ Whereas this may be considered value for money as a diagnostic management tool, the cost-benefit ratio in screening asymptomatic population groups is yet to be demonstrated. In state funded health systems, such as in the United Kingdom, these additional costs will compete with other healthcare priorities; while in some third party financed healthcare systems, a positive HSV-2 result may have implications for insurance premiums and membership of health maintenance organisations. It remains to be established if the cost-benefit ratio of population screening is as favourable, as a control measure, as what would be achieved through implementing an effective diagnostic and treatment service for symptomatic patients.

The burden and pattern of genital HSV infection vary widely across populations. General population HSV-2 prevalences are higher in the United States $(22 \%)^{8}$ than in the United Kingdom (5\%), ${ }^{9}$ as are prevalences in STD clinic attenders. The high proportion of genital disease due to HSV-1 in UK studies makes the interpretation of HSV-1 positive results in patients with no history of orolabial disease increasingly problematic. ${ }^{10}$ With the rising level of HSV-1 genital disease, a control strategy based on HSV-2 serological screening will bring diminishing returns. US neonatal herpes estimates of 20-40 cases per 100000 births $^{11}$ compare with much lower rates in European populations-1.6/100 000 live births in the United Kingdom, ${ }^{12} 6.7 / 100000$ in Sweden, ${ }^{13}$ and 4.6/100 000 in Denmark. ${ }^{14}$ In these European populations, the costbenefit ratio of screening will be much less favourable. Furthermore, even highly sensitive and highly specific tests, which are not currently widely available, will have a lower positive predictive value in low prevalence populations, ${ }^{7}$ resulting in high false positivity rates.

The case for cost effectiveness gains is based on the suggested strategy of screening women just before term to identify those at risk of acquiring genital herpes. ${ }^{12}$ Where such a woman's partner is known to have a history of genital herpes (likely to be a relatively small group of women), testing the woman late in the third trimester for HSV-1 and HSV- 2 could assist in her management, the aim of which would be to prevent transmission of the virus to the woman. In the absence of such information about the partner, a screening programme would involve the testing of all pregnant women late in the third trimester, followed by the testing of partners of all HSV-1 or HSV-2 negative women to identify which women are at risk of acquiring and subsequently transmitting infection during childbirth. Another approach - screening women late in pregnancy to identify HSV-2 seropositives - is unlikely to be effective at preventing neonatal herpes. ${ }^{15}{ }^{16}$ In the study by Brown et $a l,{ }^{16}$ none of the 94 women who seroconverted to HSV during pregnancy gave birth to a baby with neonatal herpes; and the four cases of neonatal disease, where seroconversion apparently occurred post partum, included two cases from mothers who had primary genital HSV-1 infection at term. The effectiveness and safety of suppressive therapy for women who seroconvert in the third trimester has not been established ${ }^{17}$; and the unproved benefit of active obstetric intervention where pregnant women are infected with HSV-2 suggests that screening could contribute to unnecessary caesarean sections, rather than a reduction. Third trimester screening has been recommended for regions with high incidence rates for genital or neonatal herpes. ${ }^{2}$ However, the cost effectiveness of such a strategy at different incidence rates, estimating the numbers of women to be screened, and the costs for each case averted, as well as the acceptability of such a strategy to both pregnant women and their partners, need to be assessed.

With our current state of knowledge and the diagnostic and therapeutic tools available for managing HSV-2 infections, many of the essential criteria for offering an unsolicited HSV-2 screening test, adapting these from Wilson and Jungner, ${ }^{18}$ are not yet fulfilled, particularly for the high proportion of the population which is asymptomatic: (1) the public health benefits are uncertain; (2) there is no treatment to eliminate infection; (3) the sensitivity and specificity of first generation, type specific assays and their application to low prevalence populations have yet to fully assessed; (4) the acceptability of the test to informed and 
appropriately counselled patients has not been clearly established $^{317}$; (5) there is uncertainty about its natural history-that is, the proportion of infected persons who will develop disease ${ }^{10}$; (6) there is no agreed on policy concerning who to treat; and (7) the cost-benefit and cost effectiveness of screening have not been established. In the absence of convincing evidence - that is, from randomised controlled trials which demonstrate that the benefits outweigh and justify the potential harm, the calls for widespread serological screening should be resisted.

RUAIRÍ BRUGHA

Department of Public Health and Policy, London School of Hygiene and Tropical Medicine, Keppel Street, London WC1E 7HT

Central Public Health Laboratory, 61 Colindale Avenue,

DAVID BROWN

London NW9 5 HT

ANDRÉ MEHEUS

Department of Epidemiology and Community Medicine, University of

Antwerp, Universiteitsplein 1-B-2610, Antwerp, Belgium

ADRIAN RENTON

Social Medicine and Epidemiology, Centre for Research, 200 Seagrave

Road, London SW6 1RQ

1 Ashley RL, Corey L. HSV type specific antibody tests: patients are ready, are clinicians? Genitourin Med 1997;73:235-6.

2 Corey L. Raising the consciousness for identifying and controlling viral STDS: fears and frustrations. Sex Transm Dis 1998;25:58-69.
3 Fairley I, Monteiro EF. Patient attitudes to type specific serological tests in the diagnosis of genital herpes. Genitourin Med 1997;73:259-62.

4 Vonau B, Low-Beer N, Barton SE, et al. Antenatal serum screening for genital herpes: a study of knowledge and attitudes of women at a central London hospital. Br f Obstet Gynaecol 1997;104:347-9.

5 Corey L, Ashley R, Sekulovich A, et al. Lack of efficacy of a vaccine containing recombinant $\mathrm{gD} 2$ and $\mathrm{gB} 2$ antigens in MF59 adjuvant for the prevention of genital HSV-2 acquisition. Abstract for the 37th ICACC, Toronto, Ontario, Canada.

6 Wald A. Beyond efficacy: new issues for HSV antiviral therapy. Genitourin Med 1997;73:83-4.

7 Kinghorn GR. Type-specific serological testing for herpes simplex infection. Int f STD AIDS 1998;9:497-500.

8 Fleming DT, McQuillan GM, Johnson RE, et al. Herpes simplex virus type 2 in the United States, 1976 to 1994. N Engl F Med 1997;337:1105-11.

9 Brown DWG, Vyse A, Slomka M, et al. An age stratified survey of antibody to HSV-1 and HSV-2 antibody in the UK using monoclonal antibody blocking assays. (Abstract) 12th meeting of the ISSTDR, Seville, 1997.

10 Brugha R, Keersmaekers K, Renton A, et al. Genital herpes infection: a review. Int f Epidemiol 1997;26:698-709.

11 Whitley J. Herpes simplex virus infections of women and their offspring: implications for a developed society. Proc Natl Acad Sci 1994;91:2441-7.

12 Tookey P, Peckham CS. Neonatal herpes simplex virus infections in the British Isles. Paed Perinat Epidemiol 1996;10:432-42.

13 Forsgren M, Malm G. Herpes simplex virus and pregnancy. Scand f Infect Dis Suppl 1996;100:14-19.

14 Fonnest G, de la Fuente Fonnest I, et al. Neonatal herpes in Denmark Fonnest G, de la Fuente Fonnest I, et al. Neonatal
1977-91. Acta Obstetr Gynaecol Scand 1997;76:355-8.

15 Hensleigh PA, Andrews WW, Brown Z, et al. Genital herpes during pregnancy: inability to distinguish primary and recurrent infections clinically. Obstet Gynaecol 1997;89:891-5.

16 Brown ZA, Selke S, Zeh J, et al. The acquisition of herpes simplex virus during pregnancy. N Engl Fै Med 1997;337:509-15.

17 Brocklehurst P. Antenatal serum screening for genital herpes: a study of knowledge and attitudes of women at a central London hospital. Br f Obstet Gynaecol 1997;105:125-8.

18 Wilson J, Junger G. Principles and practice of screening for disease. Geneva: World Health Organisation, Public Health Papers, 1968:34.

\section{After Bristol—how should genitourinary medicine respond?}

For many genitourinary medicine physicians the mention of Bristol will initially evoke thoughts of their recent syphilis outbreak, serving as a reminder that, like tuberculosis, the potential for the re-emergence for sexually transmitted chronic bacterial infections persists in our large cities.

However, to most clinicians, Bristol is associated with the reported failures of local self regulation in cardiothoracic surgery which has "threatened the trust which patients place in their doctors" and led to the assertion that "British medicine will be transformed" by this case. ${ }^{1}$ Certainly, the case and its sequelae have implications for all clinical disciplines. ${ }^{2}$ How will this affect genitourinary medicine?

\section{Clinical governance}

The case coincided with the new emphasis placed upon clinical governance to assure high standards of clinical care. This places obligations upon boards of NHS hospital trusts to ensure high service quality by integrating clinical audit, risk management, complaints, continuing health needs assessment, evidence based practice, and continuing professional education. This process requires that a culture of excellence is promoted, that there is clear accountability and effective clinical leadership, with clinicians and managers working together. Already, NHS hospital trusts are putting in place new structures and systems to monitor the quality of clinical practice, to ensure that clinical practice is reviewed and improved, and clinical practitioners meet standards laid down in national guidelines.

\section{Self regulation}

The Bristol case emphasised above all the failure of self regulation of doctors. Regulation of doctors is a function for which the General Medical Council (GMC) retains responsibility ${ }^{3}$ and it has been quick, as has the Royal College of Physicians (RCP), ${ }^{4}$ to make new recommendations.
It has accelerated moves to provide patients with data on the performance of their doctors and their hospitals. In order that this can be achieved, outcome data on individual treatments will need to be benchmarked so that comparisons can be made between clinicians performing the same procedures. More recently, the GMC has agreed to the introduction of new performance measures for consultants and will require that a new system is in place within 2 years.

\section{Outcome measures in genitourinary medicine}

Genitourinary medicine has an established tradition of data collection to monitor disease incidence and workload, and of participation in local, regional, and national audit projects. It differs from many other specialties, especially the surgical disciplines, in that mortality is an exceptional outcome. Nevertheless, in those clinics providing care for a large HIV cohort, a range of outcome measures can be envisaged, including mortality, and the proportions of eligible people accepting effective combination therapies. The rapid treatment advances of the past 2 years have promoted earlier diagnosis and HIV testing in genitourinary medicine clinics. We should now consider whether to collect and compare data about the proportions of newly presenting routine patients who are offered and who accept HIV testing.

Our clinical effectiveness group has produced a series of guidelines with suggested outcome measures for a wide range of genitourinary medicine conditions. Where there is a firm evidence base for these - for example, for the results of gonorrhoea and chlamydia treatment, then they may be strong contenders for inclusion in an initial package of benchmarks. Within regional audit groups, clinic specific data on disease incidence, follow up rates, and efficiency of partner notification have been collected for several years. They have revealed understandable differences in success rates between urban and more rural settings; similar inter- 
clinic and interregional differences have been apparent in British Cooperative Clinical Group studies. Nevertheless, it has been possible to gain general acceptance of a national standard based on these measures, for clinics to compare their own performance against, and to review their individual progress year on year. Meaningful outcome measures for viral sexually transmitted infections and other non-infective genital conditions have been less easy to define and will need further careful consideration.

\section{Patient assessment of clinical services}

Nevertheless, we should avoid becoming oversophisticated in our recommendations for suitable outcome measures. Too often, this approach causes some to miss the obvious. A balance must be established between ivory tower aspirations and real world attainments.

The recent CHAPS report "Quality indicators for GUM services to gay men in England," simplistic outcome measures. These consisted of:

- were you listened to?

- were you treated with respect and courtesy?

- did the clinical staff appear to know their job well?

- would you recommend that clinic to other gay men?

These are of paramount concern to our patients and vital to achieving high levels of patient acceptability. Similar consumer surveys of clinical services are to be encouraged as part of the clinical governance process.

\section{Open access and consultation time as basic quality standards}

The spread of sexually transmitted infections is favoured by factors which inhibit health seeking behaviour and prompt diagnosis both in symptomatic patients and in those who merely perceive themselves at risk of infection. One of the most important of principles of genitourinary medicine practice is that we operate an open access service. The majority of clinics continue to accept this principle, yet an increasing number have found adherence difficult, especially where workload increases have outstripped staffing resources and/or clinic facilities. Where this results in an unacceptable trade off between the quantity and quality of service provision can be easily determined by external objective audit. It should result in pressure to persuade NHS hospital trusts and health authorities of the economic folly of maintaining suboptimal services, which will inevitably lead to increasing local incidence of sexually transmitted infections and their complications and the likely spread of HIV.

Another basic quality requirement is adequate time for patient consultation. Although genitourinary medicine physicians need to acquire enhanced communication skills to establish rapport, and to educate and counsel about difficult and intensely personal issues concerning lifestyle and relationships, success has a minimum time requirement. In 1987, supported by its parent body, the RCP genitourinary medicine committee established a basic quality requirement of 20 minutes for each patient consultation which was successfully used to assess staff shortfalls (unpublished data). This argument was supported by the RCP and was unchallenged by those bodies responsible for staff level planning. A rapid expansion of consultant numbers ensued. Yet a decade later, when case complexity has increased, annual numbers of new patients continue to rise and few clinics can achieve this requirement. It will surprise no one if we confirm that those clinics with the shortest average consultation times perform least well against recommended outcome measures.

\section{External peer review and clinical audit}

In the immediate aftermath of the Bristol case, many commentators stated that the recent commercialisation of the NHS, by setting trusts in competition with each other, had resulted in economic restraints and contributed to inequalities in resources necessary to ensure uniform high quality care. It is clear that genitourinary medicine is also afflicted by these inequalities and it would be unreasonable for those who are least well off to be expected to achieve an equal performance to the better resourced. In peer audit, it is essential that structure and process measures are also included with clinical outcome measures.

As part of new recommendations, multidisciplinary clinical audit teams are being recommended for all specialties. Our experience in the Trent Health Region suggests that this is to be welcomed rather than feared. During the early 1990s, a series of annual assessments of all genitourinary medicine clinics was instituted within the region which were based upon the recommendations of the Monks report. ${ }^{6}$ The KC60 workload return provided useful supporting data about local changes in epidemiology and case mix. Each clinic was inspected by a multidisciplinary team, which usually included an administrator, a senior nurse or health adviser, a public health physician, and a genitourinary medicine physician. The teams visited each clinic for a day and looked at staffing, equipment and facilities, strategic planning, and workload/clinical outcome data. While the actual visits were not always comfortable, they were widely welcomed as they almost invariably provided support to the local clinic. The reports were sent to the NHS hospital trust chief executive with objective recommendations being made about the need for specific changes. They increased the local profile of the genitourinary medicine service and, almost invariably, were associated with resource improvements. With the demise of the regional health authorities this annual inspection process lapsed.

Many clinicians will welcome the recommendation about the need for enhanced peer review audit of clinical process and outcomes as this will update the Monks report visits we have benefited from in the past. The RCP will support this process by appointing a new college standards adviser for each trust or group of trusts who will doubtless have some involvement in the inspection process. Our responsibility will be to ensure that this individual is well informed about evidence based guidelines in our specialty.

\section{The role of service networks}

Genitourinary medicine has developed collaborative, noncompetitive, mutually supportive subregional clinic networks. Within each locality, there have been increased horizontal links with other hospital and community support and clinical disciplines, aided by the complex requirements of HIV/AIDS patient management. Vertically integrated service networks have been proposed for cancer services and this model may also be adopted for HIV/AIDS. Further enhancements to both horizontal and vertical integration of genitourinary medicine services must be considered as, beyond their core services for sexually transmitted infections management and prevention, genitourinary medicine clinics continue to diversify in their expertise. This process will be enhanced by the development of national service frameworks. More formalised networks also offer benefits to education and training and may also promote early identification of failing services and the need for their support and corrective action.

\section{Consultant appraisal}

Annual individual performance review has been a feature for non-medical staff for several years, and the progress and 
educational needs of medical trainees is regularly reviewed. Up until now consultants have been overlooked. The principles for revalidation have been established and will initially be based upon local appraisal of performance at work and participation in clinical and organisational audit. The RCP has recommended annual two way job plan review and consultant appraisal with their clinical director or medical director. Personal development plans will be supported by continuing medical education (CME). This should be welcomed for it will ensure that CME is more focused and relevant to individual need and that all consultants will be obliged to take study leave. A more difficult area concerns the identification of failing colleagues; however, the new emphasis upon support rather than judgment may help reduce the inhibitions most consultants feel about identifying colleagues in difficulty.

\section{Problems}

Many doctors understandably feel that the new clinical governance and appraisal mechanisms will merely provide the new NHS bureaucracy with "new sticks," and will do nothing to correct the underfunding of many clinics, which is the root cause of poor staff morale and impaired performance. Already, the vast majority of consultants greatly exceed their contractual commitments, want to be effective, and to actively pursue lifelong learning-but many are too exhausted to do so. The major problem will remain of having inadequate time to meet the diverse and increasingly complex demands of clinical work and administration; this will be exacerbated by the additional need to participate enthusiastically in appraisal and being appraised. If we place too great a weight upon achieving uniform high quality without a corresponding increase in resources, this will inevitably be at the expense of the available quantity of service provision. What will inevitably follow the reduced accessibility of clinic services will be the spiralling waiting lists which have blighted many other NHS specialties. For communicable diseases, this would result not only in individual distress and discomfort from physical, psychological, and relationship consequences but also in increasing spread of STI and HIV. Certainly, tinkering with service quality and regulation alone will only serve to exacerbate clinic staffing shortages and this increasing imbalance between must be addressed by politicians and administrators.

\section{Conclusion}

In the short term, a large onus will be placed upon our specialty representatives to engage in constructive and synergistic dialogue and advise upon the response to these new requirements.

The response of individual consultants will vary. The optimists will view these new requirements as being a new challenge and another opportunity to enhance our service. The pessimists will view them as yet another exercise in posture and presentation which will further jeopardise the accessibility of care. The pragmatists, including a majority of genitourinary physicians, will see that traditions of genitourinary medicine lend themselves to the new reforms and give us a lead over many other disciplines. So long as attempts to improve quality are accompanied by the necessary additional resources, especially in staffing and information technology, then a successful outcome can be assured.

\section{Royal Hallamshire Hospital, Sheffield}

G R KINGHORN

1 Smith R. All changed, utterly changed. (Editorial.) BMF 1998;316:1917-18. 2 Horton R. How should doctors respond to the GMC's judgement on Bristol? Lancet 1998;351:1900-1.

3 General Medical Council. Maintaining good medical practice. London: GMC, 1998.

4 Royal College of Physicians. Physicians maintaining good medical practice: Royal College of Physicians. Physicians maintaining good
clinical governance and self-regulation. London: RCP, 1999.

clinical governance and self-regulation. London: RCP, 1999. CHAPS. Quality indicators
Sigma Research, 1999.

6 Thin RN. Workloads in genitourinary medicine clinics in England. Genitourin Med 1989;65:376-81.

\section{Evaluating outreach clinics_are we asking too much?}

Horn and colleagues have raised an important issue for debate (see this issue, $\mathrm{p}$ 195). How do we evaluate a clinical service for sex workers linked to an outreach team? They correctly point to the limitations of the traditional method for reviewing STD services - namely, the monitoring of trends in numbers of sexually transmitted infections. The relation between STD services and STD rates is complex and disputed, particularly at the local level, and therefore monitoring incidence is usually reserved for regional or national level data. ${ }^{1}$ Useful indicators for evaluating local services should be focused on something more proximate and, indeed, measurable.

The difficulties of evaluating outreach services for marginalised groups have been discussed by Rhodes and colleagues, using the experience of the Central London Action on Street Health (CLASH), as a case study. ${ }^{2}$ At the other end of the spectrum from project evaluation, the World Health Organisation has drawn up a package of evaluation tools for national AIDS control programmes. ${ }^{34}$

Whatever the focus, it is useful to start from a broad appreciation of evaluation. Evaluation can be considered "the critical assessment, on as objective a basis as possible, of the degree to which entire services or their component parts fulfil stated goals. ${ }^{5}$ The first step is to clarify the aims of the service, and the processes undertaken to achieve them. Specific outcomes can then be defined, and methods devised to measure them.

The Edinburgh clinic described by Horn et al is linked to an outreach service and therefore combines the aim of good diagnostic and clinical care with that of health promotion, including hepatitis $B$ vaccination and effective contraception, in order to reduce morbidity and positively improve the health of local sex workers. These objectives are shared by many clinics linked to outreach services. ${ }^{6}$ Breaking down these broad aims into several limited, measurable objectives is the most difficult step in any evaluation. ${ }^{7}$ Horn and colleagues have, in contrast, adopted a more synthetic approach in which individual consultations are scored and aggregated to provide an overall score for the clinic. They have chosen to measure whether attendance was "proactive, health promoting," or "reactive, symptom driven." This approach is appealing, and relates to one of the aims of the service, which is to promote early attendance and screening where there has been potential exposure to sexually transmitted infection.

To quantify this, a system has been devised that differentiates these two ways of using the clinic, and adds weight through a set of scores. In this the activity of the clinic as a 


\section{Possible indicators for evaluation of outreach clinics}

The following ideas for evaluation are designed to be relatively easy to measure, and to be used individually or in combination to describe which aspects of the service are going well, and which need to be improved. They should be used alongside routine monitoring of clinic attendance, outreach contacts, and the profile of women contacted. As with all types of evaluation, they should be put in context by a review of the local situation, including any changes in funding, organisation, and police activity.

\section{Health protection, promotion, and proactive health behaviours}

- The proportion of patients vulnerable to hepatitis B infection at initial screen who complete a vaccination programme The proportion of patients who have backup contraception The proportion of patients who are up to date with cervical screening Proportion of cases of sexually transmitted infection (eg, chlamydia) diagnosed through asymptomatic screening Proportion of patients who know to avoid oil based lubricants with condoms

\section{Clinical care}

- The proportion of patients with a sexually transmitted infection who are treated Proportion of treated patients who return for test of cure Proportion of exposed contacts of patients with a sexually transmitted infection known to have been screened Proportion of patients with abnormal smear who are adequately followed up

\section{Outside the clinic}

- Proportion of sex workers met through outreach who are aware of the clinic Proportion of sex workers met through outreach who have used the clinic Proportion of sex workers who have been vaccinated against hepatitis B

\section{Users' views}

- No evaluation is complete without asking the users about the service, what is good, bad, and how it might be improved. This may not provide useful quantitative information, it frequently prompts changes, and can be repeated periodically to see whether changes have been effective.

whole can be summarised as aggregate rather than individual scores, and these monitored over time to see if the service is meeting the aim of shifting attendance from broadly reactive to more proactive. While appealing in its simplicity and potential reproducibility, I believe this is not the best approach to overall evaluation of their service. The fundamental problem, just like the limitation of monitoring numbers of sexually transmitted infections, is that the scores only relate to the people who attend the clinic and the relationship of them with the population served remains unclear. Since the overall objective of the service is to reduce risks and sequelae in the local sex worker population, data based only on those attending can never be a full evaluation. Also, the scoring system itself is not straightforward. The negative scoring $(-10)$ given to a termination may be appropriate if it results from a failure to use contraception; on the other hand, access to termination may be a very positive outcome for a woman who has an unwanted pregnancy due to contraceptive failure. Diagnosis of sexually transmitted infection also scores negatively $(-5)$, but this too may be the result of very appropriate health behaviour-for example, in a woman who seeks a screen soon after a burst condom.

Rather than a single, simple scoring system, I think that a number of measures have to be used in any service evaluation. Some of these can be internal, relating to the process and outcome of those who attend the clinic. Others should relate to the population served by the clinic, and be collected by the outreach team. A number of suggestions for the kind of indicators are provided in the box, and would apply to many clinical services linked to outreach and prevention work. In addition, specific indicators can be developed to relate to new initiatives. One year the Edinburgh clinic decided to promote backup contraception; this could be evaluated specifically by looking at the proportion who used any form of backup contraception and, of those who did not, what proportion were either (a) fitted for a cap, or (b) started using a hormonal contraceptive. Similarly, if the team decided to focus on emergency contraception, an evaluation may look at changes in knowledge about appropriate use and access to the "morning after pill."

Unfortunately, we have yet to identify a single, simple method of evaluating these complex services. A European perspective on evaluation, developed with the involvement of sex workers, clinicians, social workers, and researchers shows the importance of context in any evaluation. Simple measures, while appealing, are unlikely to provide an undisputed picture of the utility of a service, and should always be placed in a broader context. ${ }^{78}$

HELEN WARD

Department of Epidemiology and Public Health, Imperial College School of Medicine, St Mary's Campus, Norfolk Place, London W2 1PG

1 Renton A, Hawkes S, Hickman M, et al. Epidemiological based needs assessment: genitourinary medicine services. In: Stevens A, Raftery J, series eds. Health care needs assessment. The epidemiologically based needs assessment reviews. Second Series. Oxford: Radcliffe Medical Press Ltd, 1997:397453.

2 Rhodes T, Holland J, Hartnoll R. Hard to reach or out of reach, an evaluation of an innovative model of HIV outreach health education. London: Tuffnell Press, 1991 .

3 Mertens T, Caraël M, Sato P, et al. Editorial review: prevention indicators for evaluating the progress of national AIDS programmes. AIDS 1994;8:1359-69.

4 World Health Organisation. Evaluation of a national AIDS programme, a methods package. Geneva: WHO, 1994 (WHO/GPA/TCO/SEF 94).

5 St Leger AS, Schnieden H, Walsworth-Bell JP. Evaluating health services' effectiveness; a guide for health professionals, service managers and policy makers. Milton Keynes: Open University Press, 1992.

6 Ward H, Day S. Health care: research, services and stigma. In: Scambler G, Scambler A, eds. Rethinking prostitution. Purchasing sex in the 1990s. London: Routledge, 1997:139-64.

7 Mak R, ed. EUROPAP: European intervention projects AIDS prevention for prostitutes. Gent: Academia Press, 1996.

8 European Network for HIV/STD Prevention in Prostitution. Hustling for health: developing services for sex workers in Europe. London: Imperial College School of Medicine, 1999 (see http://www.med.ic.ac.uk/df/dfhm/europap/ index.htm). 